\title{
Analysis and Validation of Power Batteries Uniformity for Electric Vehicles
}

\author{
Li Xuling ${ }^{1, ~ a, ~ Y e ~ J i a n c h e n g ~}{ }^{1, ~ b}$, Li Zhiming ${ }^{1, c}$ \\ ${ }^{1}$ Experiment and Verification Center, State Grid Electric Power Research Institute, Nanjing, China \\ alixuling@sgepri.sgcc.com.cn, byejiancheng@sgepri.sgcc.com.cn, lizhiming@sgepri.sgcc.com.cn
}

Keywords: electric vehicle; power battery; consistency

Abstract: The technology of power batteries is an important factor on performance of EVs. In order to ensure the uniformity of the single cells, an evaluation method based on sample digital characteristic is proposed. The data from a complete charging-discharging process is collected and quantified, then the differences in charging-discharging state of single cells is obtained. The cells are divided into several classes according to the characteristic quantity as well. The method has been completed with application software. It has been applied to the test and maintenance of power batteries for electric vehicle and runs well.

\section{Introduction}

With the fast development of the Electric Vehicle, power battery has gained great attention as one of the core components in EV industry. Its technique issues about safety, reliability and lifetime have been becoming more and more notable [1-2]. In order to satisfy the requirement in service, multiple batteries have to be series connected into a group. The uniformity of single batteries in the group is a key factor that assure the lifetime [3-5]. The research on battery group uniformity, and a proper election and grouping method for batteries will make very good sense in order to improve battery management system that develop its best performance.

This paper makes an effort on analysis of reasons on battery performance uniformity, and proposed a filtering and selecting method based on the numerical feature of the sample space. This method would obtain the electric characteristics on charging-discharging performance through a quantitative evaluation. The batteries will be regrouped by the grade of the characteristic quantity. This method is evaluated and verified through experiment and mantainace in battery operations.

\section{The Uniformity of Power Batteries}

When the single batteries of same type are connected as a group, the parameters of the batteries including voltage, SOC, capacity, attenuation rate, internal resistance, lifetime and temperature have variance between each other [5]. A proper grouping method that makes the consistency of batteries as much as possible is a key issue in battery group applications [6].

Generally, the reasons that generates the variance of batteries are follows [3,7]:

(1) An initial inconsistent performance: A single battery is made with different process and material quality. The internal structure and texture has variance between each other in spite of a same batch of production. These inconsistence will becoming more series even during the service procedure.

(2) An inconsistent dynamic performance: A battery group is usually used as a mobile power source with vibrations. In such severe environment, the battery self-discharging rate will differ from each other. This would accelerate parameters inconsistence.

On the above concerns, the inconsistence in power batteries is an absolute existence. Compared in other field, the issue is much more obvious in EV applications. 


\section{The Evaluation Method on Uniformity}

This part proposed a method based on the data detected from a complete battery charging and discharging process. From the analysis and calculation, a quantitative formulation of the variance between batteries can be concluded [4,7].

Method Description

(1) The evaluation is based on the data obtained from a whole process of a battery group charging and discharging test.

(2) Calculation and analysis then would be proceeded in order to get a quantitative expression of the batteries' performance variance.

(3) A statistical approach then would be complete to get a closed solution space that satisfies a given single cell and its specifications.

\section{Assumptions}

(1) The testing data are obtained synchronously during the test including voltage U and current I.

(2) The battery group's total voltage U(t) and the voltage of a single cell would satisfy Eq.1

$$
U(t)=\sum_{i=1}^{n} U_{i}(t)
$$

The test data sequence of battery current $I(t)$ and a single cell voltage $U_{i}(t)$ can be listed as $I(m) \cdots$ and $U_{i}(m)$, where $i=1, \cdots, n$. And argument $m$ represent the sample points during the sample charging and discharging test. For a battery group composed by a number of $n$ cells, the sample space can be expressed with a matrix as Eq.2:

\section{Data Statistics}

$$
\mathrm{U}=\left(\begin{array}{c}
U_{1} \\
U_{2} \\
\vdots \\
U_{n}
\end{array}\right)=\left(\begin{array}{c}
U_{1}(m) \\
U_{2}(m) \\
\vdots \\
U_{n}(m)
\end{array}\right)=\left(\begin{array}{ccc}
u_{11} & \ldots & u_{1 m} \\
u_{21} & \ldots & u_{2 m} \\
\vdots & \ddots & \vdots \\
u_{n 1} & \ldots & u_{n m}
\end{array}\right)
$$

(1) Firstly, get the calculation of the Pearson factor $\gamma_{k l}$ between any two single cells (assuming cell k\# and cell $1 \#$ ) from the data $U_{i}(t)$ during charging and discharging. The calculation formula is shown as Eq.3:

$$
\gamma_{k l}=\frac{\operatorname{COV}\left(U_{k}, U_{l}\right)}{\sqrt{D U_{k}} \sqrt{D U_{l}}}
$$

(2) From the formula above, we derive the Pearson correlation coefficient matrix as Eq.4:

$$
\gamma=\left(\begin{array}{rll}
\gamma_{11} & \ldots & \gamma_{1 n} \\
\gamma_{21} & \ldots & \gamma_{2 n} \\
\vdots & \ddots & \vdots \\
\gamma_{n 1} & \ldots & \gamma_{n n}
\end{array}\right)
$$

where $\gamma_{j n}=\gamma_{n j}$ and $\gamma_{j j}=1$.

From the correlation coefficient we introduce a non-dimensional argument $\alpha$ that satisfies Eq.5:

$$
\alpha_{i}=E\left(\gamma_{i j}\right)
$$

where $i=1,2, \cdots, n$. The factor $\alpha_{i}$ represents the level of the average correlation variance between cell i\# and other cells.

\section{Evaluation Method}

When $\alpha_{i}>0.8$, the two cells are highly correlated and have good uniformity. It is recommended to assemble them in one group. 
When $0.5<\alpha_{i}<0.8$, the two cells are fairly correlated. It is not recommended to assemble them in one group.

When $0.3<\alpha_{i}<0.5$, the two cells are lowly correlated. It is not in a condition for assembling and they should be abandoned.

When $\alpha_{i}<0.3$, the two cells are barely correlated. They must be abandoned and replaced by new ones.

\section{Applications}

\section{Experiment Environment}

In this research, an 180Ah power battery group made up by 16 series connected single cells is tested in by constant current charging. The specific testing equipment is a Chroma-17020 designed for testing power batteries. When testing is started, a rated constant current is set in order to charge the battery until the charging is stopped and meet the cut-off condition.

During the charging process, the charging current $I(t)$, voltage of single cells $U_{i}(t)$ are recorded as $I(m)$ and $U_{i}(m)$, where $i=1, \cdots, 16$. Argument $m$ is the number of recorded voltage during charging. Evaluations

According to Eq. 2 the testing voltage data for cell i\# is $U_{i}(m)=\left\{u_{i 1}, u_{i 2}, \ldots, u_{i m}\right\}$. The capacity of the battery group can be calculated with Eq.6:

$$
\mathrm{C}_{\text {Batteries }}(I)=\sum_{k=0}^{m} A_{k} I(k)
$$

where

$$
\begin{gathered}
A_{k}=\int_{t_{0}}^{t_{s}} l_{k}(t) d t \\
l_{k}(t)=\prod_{\substack{j=0 \\
j \neq k}}^{m} \frac{t-t_{j}}{t_{k-} t_{j}}
\end{gathered}
$$

As a result, the curve of voltage-capacity can be got in the Battery Testing and Maintenance System software as shown in Fig.1.

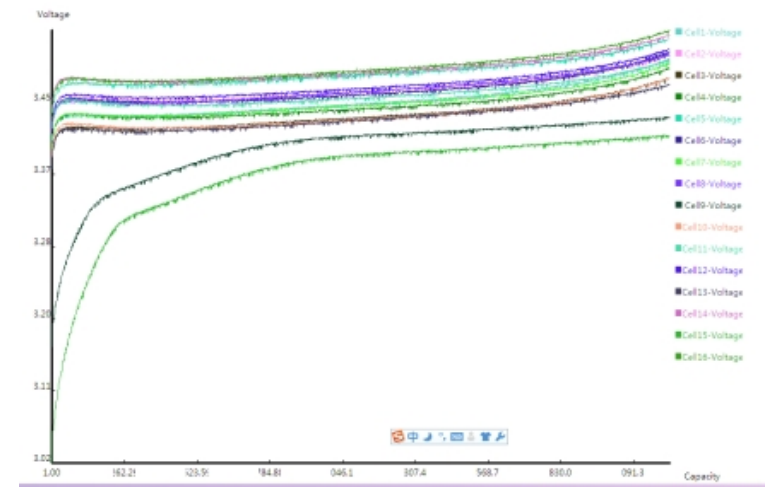

Fig. $12 \mathrm{D}$ curves of Voltage-Capacity

According to the statistic method introduced above, the Pearson coefficient can be get as shown in Fig.2. Data in red font are the batteries with obviously low correlation. 


\begin{tabular}{|c|c|c|c|c|c|c|c|c|c|c|c|c|c|c|c|c|}
\hline & Cell1 & Cell2 & Cell3 & Cell4 & Cell5 & Cell6 & Cell & Cells & Cell19 & Cell10 & Cell11 & Cell12 & Cell13 & Cell14 & Cell15 & Cell16 \\
\hline Cell1 & 1.00 & 0.99 & 0.99 & 0.99 & 0.99 & 0.99 & 0.99 & 0.99 & 0.69 & 0.99 & 0.99 & 0.99 & 0.99 & 0.99 & 0.64 & 0.99 \\
\hline Cell2 & 0.99 & 1.00 & 0.99 & 0.99 & 0.99 & 0.99 & 0.99 & 0.99 & 0.66 & 0.99 & 0.99 & 0.99 & 0.99 & 0.99 & 0.60 & 0.99 \\
\hline Cell3 & 0.99 & 0.99 & 1.00 & 0.99 & 0.99 & 0.99 & 0.99 & 0.99 & 0.70 & 0.99 & 0.99 & 0.99 & 0.99 & 0.99 & 0.65 & 0.99 \\
\hline Cell4 & 0.99 & 0.99 & 0.99 & 1.00 & 0.99 & 0.99 & 0.99 & 0.99 & 0.64 & 0.99 & 0.99 & 0.99 & 0.99 & 0.99 & 0.59 & 0.99 \\
\hline Cell5 & 0.99 & 0.99 & 0.99 & 0.99 & 1.00 & 0.99 & 0.99 & 0.99 & 0.70 & 0.99 & 0.99 & 0.99 & 0.99 & 0.99 & 0.65 & 0.99 \\
\hline Cell6 & 0.99 & 0.99 & 0.99 & 0.99 & 0.99 & 1.00 & 0.99 & 0.99 & 0.62 & 0.99 & 0.99 & 0.99 & 0.99 & 0.99 & 0.57 & 0.99 \\
\hline Cell7 & 0.99 & 0.99 & 0.99 & 0.99 & 0.99 & 0.99 & 1.00 & 0.99 & 0.67 & 0.99 & 0.99 & 0.99 & 0.99 & 0.99 & 0.63 & 0.99 \\
\hline Cells & 0.99 & 0.99 & 0.99 & 0.99 & 0.99 & 0.99 & & 1.00 & 0.69 & 0.99 & 0.99 & 0.99 & 0.99 & 0.99 & 0.64 & 0.99 \\
\hline Cell9 & 0.69 & 0.66 & 0.70 & 0.64 & 0.70 & 0.62 & 0.67 & 0.69 & 1.00 & 0.63 & 0.60 & 0.64 & 0.99 & 0.68 & 0.99 & 0.68 \\
\hline Cell10 & 0.99 & 0.99 & 0.99 & 0.99 & 0.99 & 0.99 & 0.99 & 0.99 & 0.63 & 1.00 & 0.99 & 0.99 & 0.99 & 0.99 & 0.58 & 0.99 \\
\hline Cell11 & 0.99 & 0.99 & 0.99 & 0.99 & 0.99 & 0.99 & 0.99 & 0.99 & 0.60 & 0.99 & 1.00 & 0.99 & 0.99 & 0.99 & 0.55 & 0.99 \\
\hline Cell12 & 0.99 & 0.99 & 0.99 & 0.99 & 0.99 & 0.99 & 0.99 & 0.99 & 0.64 & 0.99 & 0.99 & 1.00 & 0.99 & 0.99 & 0.61 & 0.99 \\
\hline Cell13 & 0.99 & 0.99 & 0.99 & 0.99 & 0.99 & 0.99 & 0.99 & 0.99 & 0.99 & 0.99 & 0.99 & 0.99 & 1.00 & 0.99 & 0.65 & 0.99 \\
\hline Cell14 & 0.99 & 0.99 & 0.99 & 0.99 & 0.99 & 0.99 & 0.99 & 0.99 & 0.68 & 0.99 & 0.99 & 0.99 & 0.99 & 1.00 & 0.59 & 0.99 \\
\hline Cell15 & 0.64 & 0.60 & 0.65 & 0.59 & 0.65 & 0.57 & 0.63 & 0.64 & 0.99 & 0.58 & 0.55 & 0.61 & 0.65 & 0.59 & 1.00 & 0.63 \\
\hline Cell16 & 0.99 & 0.99 & 0.99 & 0.99 & 0.99 & 0.99 & 0.99 & 0.99 & 0.68 & 0.99 & 0.99 & 0.99 & 0.99 & 0.99 & 0.63 & 1.00 \\
\hline
\end{tabular}

Fig.2 Pearson Correlation Coefficient

In order to describe the correlation situation in a much more visual way, the scatter diagram of the data can be drawn in Fig.3. It is easy to see that cell 9\# and cell 15\# (with a S or reversed S curve) have a much lower Pearson correlation coenfficient compared with other batteries.

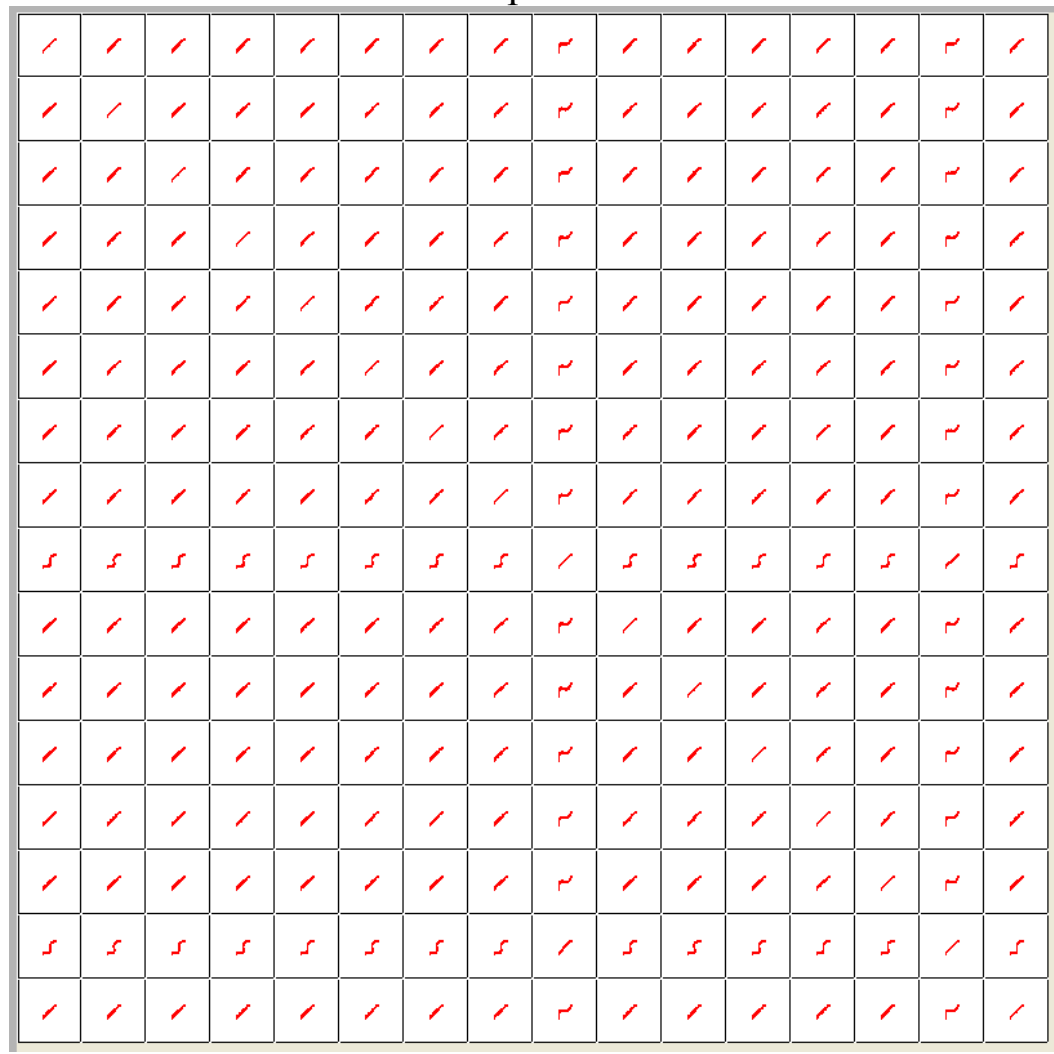

Fig. 3 Scatter Diagram

According to Eq.5 and Eq.6, the value can be calculated in Fig.4. 


\begin{tabular}{|l|c|}
\hline & $\alpha_{\mathrm{i}}$ \\
\hline Cell1 & 0.9501 \\
\hline Cell2 & 0.9475 \\
\hline Cell3 & 0.9529 \\
\hline Cell4 & 0.9463 \\
\hline Cell5 & 0.9531 \\
\hline Cell6 & 0.9422 \\
\hline Cell1 & 0.952 \\
\hline Cell8 & 0.9521 \\
\hline Cell1 & 0.7042 \\
\hline Cell10 & 0.9443 \\
\hline Cell11 & 0.9379 \\
\hline Cell12 & 0.9492 \\
\hline Cell13 & 0.9519 \\
\hline Cell14 & 0.9456 \\
\hline Cell15 & 0.6616 \\
\hline Cell16 & 0.9515 \\
\hline
\end{tabular}

Fig.4 Value of $\alpha_{i}$

According to the evaluation and assembling method disscussed above, the cell $9 \#$ and cell $15 \#$ should be replaced. In the pratical experiment, cell 9\# and cell 15\# are the fastest to reach the charging saturated voltagte and the whole group charging is stopped as a result. The total capacity is wasted due to the saturation. So the experiment is agreed with the theory analysis.

It is notable to say that the parameter is determined according to the statistac method and recommended as a general indicated value. In a pratical application, the specifications should be increased or reduced accroding to the requirement.

\section{Summary}

In this paper, the importance of the uniformity for the power battery is introduced. An evaluation and grouping method for the batteries is researched and verified. At present, this method is successfully applied in EV power battery group testing and maintenance. The process shows very fast and effective in the practical applications.

\section{Acknowledgements}

This work was financially supported by the National High Technology Research and Development $\operatorname{Pr}$ ogram (863Program) (2011AA05A110).

\section{References}

[1] Zechun $\mathrm{Hu}$, Yonghua Song, Zhiwei $\mathrm{Xu}$, et al. Impacts and Utilization of Electric Vehicles Integration Into Power Systems. Proceedings of CSEE. 2012(4):1-10.

[2] Yiqun Wang, Liang Chen, Yingjie Bai, et al. Domestic Standards Situation of Lithium-ion Traction Battery for Electric Vehicles. Standard Science. 2012(7):26-28.

[3] Zhenpo Wang, Fengchun Sun, Chenan Lin. Analysis on the Influence of Inconsistencies upon the Service Life of Power Battery Packs. Transactions of Beijing Institute of Technology. 2006(07):577.

[4] Zhenpo Wang, Fengchun Sun, Chengning Zhang. Study on inconsistency of electric vehicle battery pack. Chinese Journal of Power Sources. 2003(05):438.

[5] Youliang Ma, Quanshi Chen. The Inconsistent Influence Analysis of Battery for Hybrid Electric Vehicle. Auto Electric Parts. 2001(02):5.

[6] Jiayuan Wang, Zechang Sun, Xuezhe Wei, et al. Lifespan Uniformity for the Battery Pack in the Automotive Application. Journal of Automotive Safety and Energy. 2011(03):223.

[7] Chao Chen. Design and Application of Lithium-ion Power Battery Test System: Master Dissertation. Zhejiang University. 2011 